\title{
Radiation dose of cardiac computed tomography - what has been achieved and what needs to be done
}

\author{
Hatem Alkadhi, MD, MPH • Sebastian Leschka, MD
}

Received: 2 August 2010 /Revised: 27 August 2010 / Accepted: 10 September 2010/Published online: 19 October 2010

(C) European Society of Radiology 2010

\begin{abstract}
This review highlights the recently introduced techniques by manufacturers and various research workers to reduce radiation dose in coronary $\mathrm{CT}$. It discusses in detail the development of ECG-based tube current modulation, the application of low tube voltage protocols and prospective ECG-gating. It also briefly discusses two further methods of dose reduction, namely minimisation of the $x-y$ anatomical coverage and adaptive statistical iterative reconstruction.
\end{abstract}

\section{Introduction}

In the October issue of European Radiology in 2009, Ertel and colleagues [1] described a spiral computed tomography (CT) technique employing a very high table feed (and pitch) for a fast data acquisition of the heart. The authors performed experiments in three pigs using a first-generation dual-source CT machine equipped with prototype software allowing pitch factors up to 3.0. The high-pitch mode delivered good image quality of the pig hearts, similar to that obtained from a standard spiral low-pitch data acquisition technique. Most importantly, the corresponding effective radiation dose of a high-pitch cardiac CT study in their first experiments was only $2.0 \mathrm{mSv}$.

H. Alkadhi MD, MPH $(\bowtie)$

Institute of Diagnostic and Interventional Radiology,

University Hospital Zurich,

Raemistr. 100,

8091 Zurich, Switzerland

e-mail: hatem.alkadhi@usz.ch

S. Leschka MD

Institute of Radiology, General Hospital,

Saint Gall, Switzerland
In the November issue of European Radiology in 2009, Lell and colleagues [2] could extend these initial results to a first feasibility study employing the high-pitch mode in a series of 25 patients and using a second-generation dualsource CT machine. Data were acquired synchronized to the electrocardiogram (ECG), and starting at $60 \%$ of the R$\mathrm{R}$ interval with a pitch of 3.2 or 3.4 .

Again, image quality of the heart and coronary arteries using the high-pitch mode was good to excellent, along with a very small rate of segments having a non-diagnostic image quality. Importantly, the average estimated effective radiation dose of the high-pitch mode was $0.88 \mathrm{mSv}$ and $1.0 \mathrm{mSv}$, depending on the protocol used.

These two studies nicely illustrate the most recent achievement in radiation dose lowering algorithms, enabling a cardiac CT study for the non-invasive evaluation of the coronary arteries with a radiation dose of around (or even below) $1 \mathrm{mSv}$. Confirmation of these results was published thereafter in a number of studies [3, 4], also demonstrating the high diagnostic performance of the highpitch mode for coronary imaging when compared to the reference standard modality catheter coronary angiography $[5,6]$. In this editorial, we want to briefly summarize and highlight the most amazing achievements that were made over the last few years in regard to the radiation dose of cardiac $\mathrm{CT}$, resulting in a tremendous reduction in radiation exposure to the patients undergoing this non-invasive imaging test.

\section{Summary of radiation dose reducing techniques}

The recent years showed an overall increase in the use of $\mathrm{CT}$ for imaging of the heart and coronary arteries. For example in the United States, around 2.3 million CT 
coronary angiography examinations are currently performed annually [7]. The downside of this increased use of the radiation-associated cardiac imaging test is the increase in the collective radiation dose, which might pose a risk to the population [8]. It is obvious that the strategy to lower the overall radiation burden to the population must be twofold: First, the radiation dose of the individual CT study should be lowered to a level that is as low as reasonably achievable; and second, the total number of CT studies must be controlled by strict adherence to common guidelines and recommendations.

With ECG-gated cardiac CT, radiation dose is closely related to the table feet, or pitch value. The pitch for spiral CT coronary angiography studies as e.g. employed with 64slice CT machines is typically low, ranging from 0.2 to 0.5 . This indicates that the table is advanced by less than one detector width during each gantry rotation. Thus, this technique exposes the same anatomic area to X-ray radiation during consecutive rotations of the gantry, which results in a relatively high radiation dose. With retrospectively ECG-gated cardiac single-source 64-slice CT, radiation dose values of up to $21 \mathrm{mSv}$ have been reported for coronary imaging [9], while average effective doses of this technique are in the range of $15 \mathrm{mSv}$ [8, 10-12] (Fig. 1).

\section{ECG-based tube current modulation}

An important approach for lowering the dose of retrospectively ECG-gated cardiac CT is ECG-based tube current modulation. With this technique, the tube current outside the diastole is lowered to $25 \%$ of the nominal value. Thus, the effective radiation dose of retrospectively ECG-gated CT coronary angiography can be considerably lowered, with a drop of effective dose to around $10 \mathrm{mSv}$ [10-14] (see Fig. 1).

Another approach for radiation dose reduction with retrospective ECG-gating is MinDose (Siemens Healthcare), representing a technique that lowers the tube current outside the diastole to only $4 \%$ of the nominal value [15]. With this algorithm, which is particularly efficient at lower heart rates, the radiation dose of a CT coronary angiography study can be further lowered down to around $8 \mathrm{mSv}$ (see Fig. 1).

\section{Low tube voltage}

The next step in reducing radiation dose of CT coronary angiography was the application of low tube voltage protocols [16, 17]. Lowering the tube voltage represents an important radiation dose reduction approach because the radiation dose varies with the square of the tube voltage [18]. Lowering the tube voltage has the additional advantage of the higher attenuation levels for iodinated contrast medium as a result of a greater photoelectric effect and decreased Compton scattering [16]. This effect can be also used for lowering the contrast medium volume while obtaining the same attenuation within the vessels. With reduction of the tube voltage from the standard $120 \mathrm{kV}$ to $100 \mathrm{kV}$ (Fig. 2a), another lowering of the average effective radiation dose of retrospectively ECG-gated cardiac CT with ECG-controlled tube current modulation to around $6.5 \mathrm{mSv}$ was achieved [16, 17] (see Fig. 1). It must be noted, however, that lowering of the tube voltage should be done according to the body physiognomy of the patient (e. g. only in patients with a body mass index below $25 \mathrm{~kg} / \mathrm{m}^{2}$ [16]).

\section{Prospective ECG-gating}

The next quantum leap in radiation dose reduction of cardiac CT was the reintroduction of prospective ECGgating, or the step-and-shoot mode. Prospective ECGgating is characterized by turning on the X-ray tube only at a predefined time point of the cardiac cycle, usually in diastole, while keeping the patient table stationary. The $\mathrm{X}$ ray exposure time of this technique is short, and thus, low radiation doses ranging between 1.5 to $4 \mathrm{mSv}$ have been reported with this technique, depending on the use of a standard $120 \mathrm{kV}$ or a low tube voltage protocol at $100 \mathrm{kV}$ [19] (see Fig. 1). Importantly, this radiation dose saving technique can be reliably and accurately employed with various $\mathrm{CT}$ machines, including single-source and dualsource CT scanners, as well as broader detector systems with 256- or 320-slices [20-24] (Fig. 2b). With this approach, cardiac CT can be even used for the accurate diagnosis of congenital heart disease in infants and children [25]. Of note, however, is the requirement of a stable sinus rhythm with a relatively low heart rate (i.e., below 65 or $70 \mathrm{bpm}$ ) for being eligible for this technique.

\section{ECG-gated high-pitch mode}

One of the most recent advents in radiation dose saving algorithms for cardiac CT is - as discussed already above $[1,2]$ - the ECG-gated high-pitch mode (Fig. 2c). With this mode, data is acquired in a spiral mode while the table runs with a high pitch of maximally 3.4 , equaling a table feed of $46 \mathrm{~cm} / \mathrm{s}$. When using the high-pitch mode, the entire heart can be scanned within one single cardiac cycle, usually during diastole. The effective radiation dose of the highpitch mode for a cardiac CT study is as low as $1 \mathrm{mSv}$ [3-6] (see Fig. 1), and around 2 to $4 \mathrm{mSv}$ for an ECG-gated CT angiography study of the entire chest $[3,26]$. This can be explained by the fact that using high-pitch imaging, 'superfluous' X-rays exposing the entire width of the detector as it enters and leaves the entire arc of projections 
Fig. 1 Bar graph illustrating the average effective radiation doses of cardiac CT applying the various radiation dose reducing algorithms

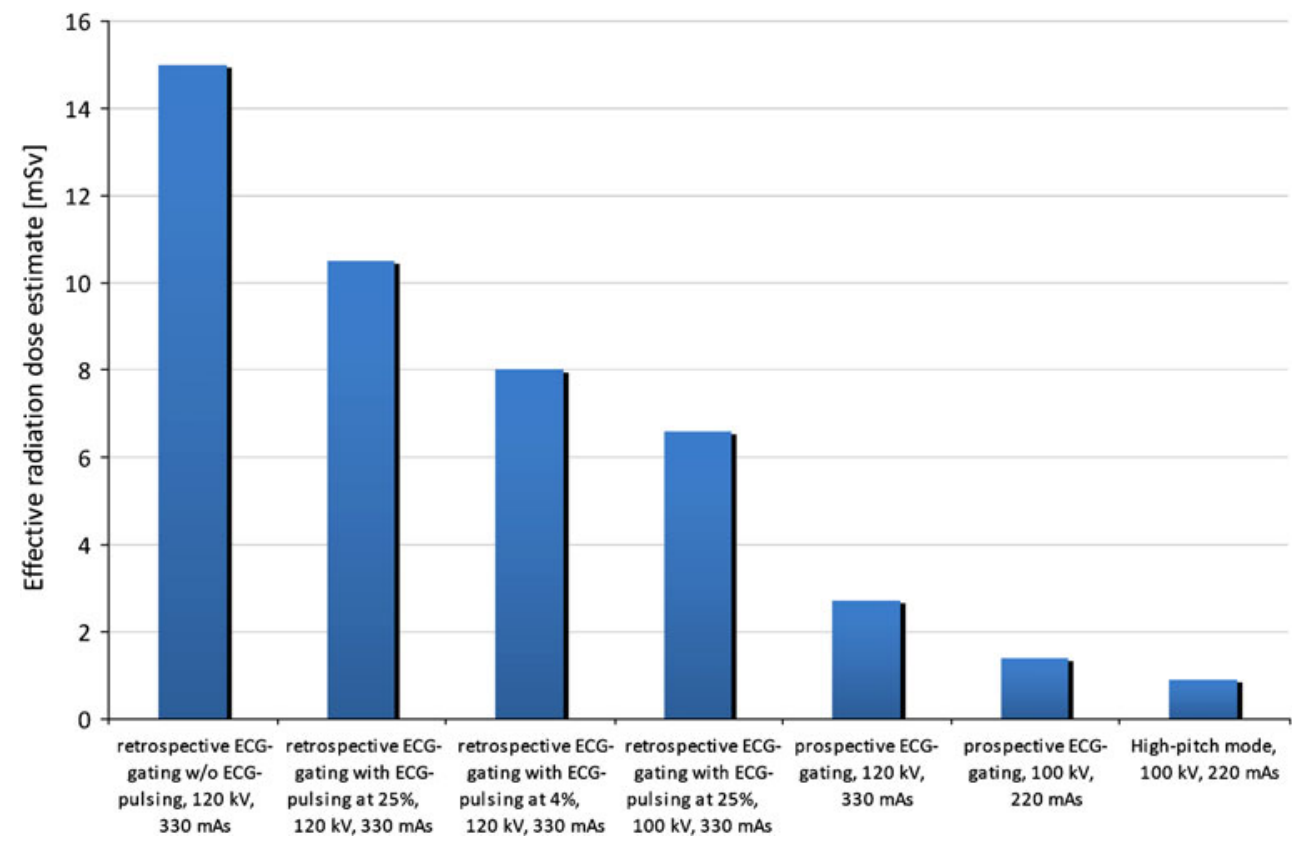

are applied only once at the beginning and end of the spiral path $[1,2,6]$. With prospective ECG-gating (or step-andshoot mode), such 'superfluous' exposure occurs for each acquired slice, which explains the further reduction of radiation dose to the patient with the high-pitch mode. Again, it must be noted, that a stable sinus rhythm with a relatively low heart rate (i.e., below $63 \mathrm{bpm}$ ) is required for being eligible for this technique.

\section{Additional means to lower the radiation dose}

Various additional means exist to further lower the radiation dose of cardiac CT. Among these, limiting the cranio- caudal coverage length, use of bowtie filters, and statistical iterative reconstruction algorithms will be highlighted in this brief review. Of note, these radiation reducing approaches can be used in addition to the dose-saving algorithms outlined above, which will result in additional lowering of the radiation burden to the patient.

The region undergoing radiation exposure in the craniocaudal is the largest contributor to radiation absorption. The CT scout (or topogram) is quite inaccurate for determining the precise extent of the coronary arteries. An alternative is to use images from the coronary calcium scoring to set the upper limit above the apex of the left anterior descending artery and the lower limit inferior to the posterior descend-
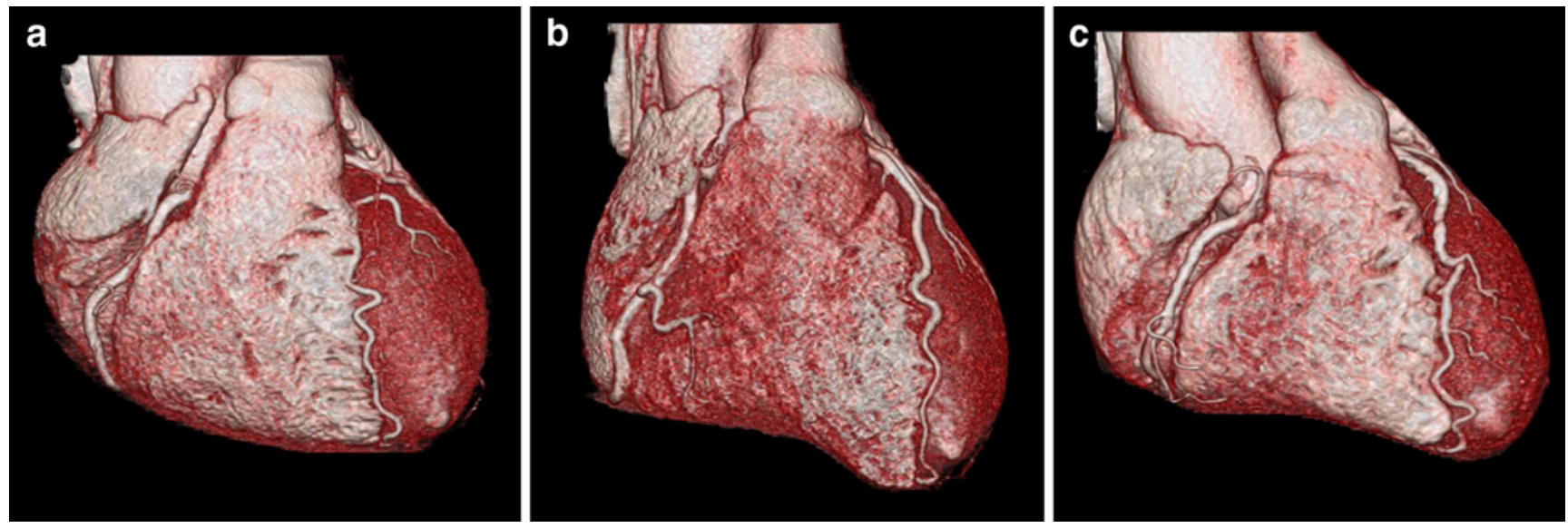

Fig. 2 Examples of image quality and radiation exposure with different cardiac CT protocols. (a) 65-year-old woman with a BMI of $22.1 \mathrm{~kg} / \mathrm{m}^{2}$ and a heart rate of $81 \mathrm{bpm}$. CT was performed using retrospective ECG-gating with ECG-pulsing at a full tube current window from $30-80 \%$ of the R-R interval and a tube voltage of $100 \mathrm{kV}$. Radiation dose estimate was $6.2 \mathrm{mSv}$. (b) 55 -year-old man with a BMI of $28.0 \mathrm{~kg} / \mathrm{m}^{2}$ and a heart rate of $70 \mathrm{bpm}$. CT was performed using prospective ECG-gating and a tube voltage of $120 \mathrm{kV}$. Radiation dose estimate was $2.5 \mathrm{mSv}$. (c) 51-year-old man with a BMI of $23.9 \mathrm{~kg} / \mathrm{m}^{2}$ and a heart rate of $61 \mathrm{bpm}$. CT was performed using a high-pitch CT protocol at a tube voltage of $100 \mathrm{kV}$. Radiation dose estimate was $1 \mathrm{mSv}$ 
ing artery, leaving sufficient margins to allow for movement [27]. This simple approach helps to further reduce the radiation dose by about $16 \%$ [28].

Another technique that might be underused is to restrict the $x-y$ field of view. Bowtie filters allow for smaller radiation exposure by limiting the scatter of the X-ray towards the detectors. Thus, bowtie filters allow less X-rays out of the central part of the X-ray tube, as less are required to expose the detectors. Use of bowtie filters have potential to reduce the radiation exposure to the patient by up to $40 \%$ [27].

Statistical iterative reconstruction algorithms are an alternative to the classic reconstruction technique of $\mathrm{CT}$, $\mathrm{i}$. e., filtered back projection. This technique incorporates statistical modelling to reduce image noise, which may permit preserved image quality with reduced tube current, thereby permitting lower radiation dose. As a matter of fact, first experience with statistical iterative reconstruction for coronary artery imaging has shown a $44 \%$ reduction in the median radiation dose when compared to CT protocols using filtered back projection for image reconstruction [29].

\section{Outlook and conclusion}

This brief overview was intended to demonstrate and highlight the many steps that were undertaken in the past years for lowering the radiation exposure to patients undergoing cardiac CT. Employing these techniques, the effective radiation dose of a CT coronary angiography study can be reduced from around 15 to as low as $1 \mathrm{mSv}$ in selected patients. It is important to note that the current effective radiation doses from cardiac CT are at the level or even below those reported for the reference standard modality catheter coronary angiography. In addition, it is also lower than that of a standard chest or abdominal CT study. Thus, the achievements in radiation dose reduction in cardiac CT must be considered tremendous, and similar success stories in radiation dose lowering techniques for CT of other body regions are still be awaited.

Nevertheless, still a number of issues and problems need to be addressed and solved in the near future. Considerable problems continue to be present for the CT imaging of coronary artery stents. While CT stent imaging can be now performed - similar to the imaging of the native coronary arteries - at a low radiation dose, the in-stent visualization still is limited [30, 31], and a routine application of CT for the follow-up of coronary stent patency cannot be recommended so far.

Another important issues is the question on how we will assure that all these radiation dose lowering protocols for cardiac CT are used by the increasingly large radiological community performing cardiac imaging. In parallel, cardiac imagers must strictly adhere to the appropriate indications for cardiac CT. For example, use of this modality in asymptomatic subjects for screening purposes (as part of so-called check-up examinations) should be disallowed. Otherwise, all our efforts to lower the radiation dose of individual CT studies will be thwarted by an increasing total number of imaging tests. When the latter cannot be prevented, the collective radiation dose to the population undergoing X-ray-based cardiac imaging might continue to increase even further.

\section{References}

1. Ertel D, Lell MM, Harig F et al (2009) Cardiac spiral dual-source CT with high pitch: a feasibility study. Eur Radiol 19:2357-2362

2. Lell M, Marwan M, Schepis T et al (2009) Prospectively ECGtriggered high-pitch spiral acquisition for coronary $\mathrm{CT}$ angiography using dual source CT: technique and initial experience. Eur Radiol 19:2576-2583

3. Sommer WH, Schenzle JC, Becker CR et al (2009) Saving dose in triple-rule-out computed tomography examination using a highpitch dual spiral technique. Invest Radiol 45:64-71

4. Lell M, Hinkmann F, Anders K et al (2009) High-pitch electrocardiogram-triggered computed tomography of the chest: initial results. Invest Radiol 44:728-733

5. Leschka S, Stolzmann P, Desbiolles L et al (2009) Diagnostic accuracy of high-pitch dual-source CT for the assessment of coronary stenoses: first experience. Eur Radiol 19:2896-2903

6. Alkadhi H, Stolzmann P, Desbiolles L et al (2010) Low-dose, 128-slice, dual-source CT coronary angiography: accuracy and radiation dose of the high-pitch and the step-and-shoot mode. Heart 96:933-938

7. Mettler FA Jr, Bhargavan M, Faulkner K, Gilley DB, Gray JE, Ibbott GS, Lipoti JA, Mahesh M, McCrohan JL, Stabin MG, Thomadsen BR, Yoshizumi TT (2009) Radiologic and nuclear medicine studies in the United States and worldwide: frequency, radiation dose, and comparison with other radiation sources1950-2007. Radiology 253(2):520-531

8. Alkadhi H (2009) Radiation dose of cardiac CT-what is the evidence? Eur Radiol 19:1311-1315

9. Mollet NR, Cademartiri F, van Mieghem CA et al (2005) Highresolution spiral computed tomography coronary angiography in patients referred for diagnostic conventional coronary angiography. Circulation 112:2318-2323

10. Hausleiter J, Meyer T, Hadamitzky M et al (2006) Radiation dose estimates from cardiac multislice computed tomography in daily practice: impact of different scanning protocols on effective dose estimates. Circulation 113:1305-1310

11. Alkadhi H, Stolzmann P, Scheffel H et al (2008) Radiation dose of cardiac dual-source CT: the effect of tailoring the protocol to patient-specific parameters. Eur J Radiol 68:385-391

12. Achenbach S, Anders K, Kalender WA (2008) Dual-source cardiac computed tomography: image quality and dose considerations. Eur Radiol 18:1188-1198

13. Jakobs TF, Becker CR, Ohnesorge B et al (2002) Multislice helical CT of the heart with retrospective ECG gating: reduction of radiation exposure by ECG-controlled tube current modulation. Eur Radiol 12:1081-1086

14. Stolzmann P, Scheffel H, Schertler T et al (2008) Radiation dose estimates in dual-source computed tomography coronary angiography. Eur Radiol 18:592-599 
15. Pflederer T, Jakstat J, Marwan M et al (2010) Radiation exposure and image quality in staged low-dose protocols for coronary dualsource CT angiography: a randomized comparison. Eur Radiol 20:1197-1206

16. Leschka S, Stolzmann P, Schmid FT et al (2008) Low kilovoltage cardiac dual-source CT: attenuation, noise, and radiation dose. Eur Radiol 18:1809-1817

17. Paul JF, Abada HT (2007) Strategies for reduction of radiation dose in cardiac multislice CT. Eur Radiol 17:2028-2037

18. Huda W, Scalzetti EM, Levin G (2000) Technique factors and image quality as functions of patient weight at abdominal CT. Radiology 217:430-435

19. Lehmkuhl L, Gosch D, Nagel HD et al (2010) Quantification of radiation dose savings in cardiac computed tomography using prospectively triggered mode and ECG pulsing: a phantom study. Eur Radiol 20:2116-2125

20. Klass O, Jeltsch M, Feuerlein S et al (2009) Prospectively gated axial CT coronary angiography: preliminary experiences with a novel low-dose technique. Eur Radiol 19:829-836

21. Herzog BA, Wyss CA, Husmann L et al (2009) First head-to-head comparison of effective radiation dose from low-dose 64-slice CT with prospective ECG-triggering versus invasive coronary angiography. Heart 95:1656-1661

22. Scheffel H, Alkadhi H, Leschka S et al (2008) Low-dose CT coronary angiography in the step-and-shoot mode: diagnostic performance. Heart 94:1132-1137

23. Klass O, Walker M, Siebach A et al (2010) Prospectively gated axial CT coronary angiography: comparison of image quality and effective radiation dose between 64- and 256-slice CT. Eur Radiol 20:1124-1131
24. Dewey M, Zimmermann E, Deissenrieder F et al (2009) Noninvasive coronary angiography by 320 -row computed tomography with lower radiation exposure and maintained diagnostic accuracy: comparison of results with cardiac catheterization in a head-to-head pilot investigation. Circulation 120:867-875

25. Cheng Z, Wang X, Duan Y et al (2010) Low-dose prospective ECG-triggering dual-source CT angiography in infants and children with complex congenital heart disease: first experience. Eur Radiol 20(10):2503-2511

26. Goetti R, Leschka S, Baumuller S et al (2010) Low dose highpitch spiral acquisition 128-slice dual-source computed tomography for the evaluation of coronary artery bypass graft patency. Invest Radiol 45:324-330

27. Budoff MJ (2009) Radiation dose of cardiac CT-what is the evidence? Int J Cardiovasc Imaging 25:279-287

28. Leschka S, Kim CH, Baumueller S et al (2010) Scan length adjustment of CT coronary angiography using the calcium scoring scan: effect on radiation dose. AJR 194:W272-W277

29. Leipsic J, Labounty TM, Heilbron B et al (2010) Estimated radiation dose reduction using adaptive statistical iterative reconstruction in coronary CT angiography: the ERASIR study. AJR 195:655-660

30. Horiguchi J, Fujioka C, Kiguchi M et al (2009) Prospective ECGtriggered axial $\mathrm{CT}$ at $140-\mathrm{kV}$ tube voltage improves coronary instent restenosis visibility at a lower radiation dose compared with conventional retrospective ECG-gated helical CT. Eur Radiol 19:2363-2372

31. Wolf F, Leschka S, Loewe C et al (2010) Coronary artery stent imaging with 128-slice dual-source CT using high-pitch spiral acquisition in a cardiac phantom: comparison with the sequential and low-pitch spiral mode. Eur Radiol 20:2084-2091 